\title{
Current Management of Hemobilia
}

\author{
Victor M. Zaydfudim • John F. Angle • \\ Reid B. Adams
}

Published online: 5 April 2014

(C) Springer Science + Business Media New York 2014

\begin{abstract}
Hemobilia is an uncommon source of significant gastrointestinal bleeding resulting from a communication between the vascular and biliary systems. Iatrogenic injury to the liver and the biliary system from percutaneous transhepatic techniques is currently the leading cause of hemobilia. Trauma, operative injury, malignancy, and inflammatory diseases of the hepatobiliary system are other rare, but important, etiologies for clinically significant hemobilia. While diagnostic CT angiography can be used in clinically stable patients, interventional angiography and transcatheter percutaneous endovascular techniques are the standard for diagnosis and treatment of hemobilia. Operative intervention is not required for management in the vast majority of the patients. Appropriate biliary drainage must be ensured after bleeding is addressed.
\end{abstract}

Keywords Hemobilia - Interventional radiology · Embolization · Trans-catheter therapy · Endovascular . Minimally invasive technique $\cdot$ Management

This article is part of the Topical Collection on Acute Care Surgery.

V. M. Zaydfudim · R. B. Adams ( $\square)$

Department of Surgery, University of Virginia, Box 800709,

Charlottesville, VA 22908, USA

e-mail: rba3b@virginia.edu

V. M. Zaydfudim

e-mail: vz8h@virginia.edu

J. F. Angle

Department of Radiology, University of Virginia, Box 800170,

Charlottesville, VA 22908, USA

e-mail: jfa3h@virginia.edu

\section{Introduction}

Hemobilia is an uncommon source of bleeding resulting from a communication between the vascular and biliary systems. While it was originally described in 1654 by Francis Glisson as a cause of death in a patient after a penetrating liver injury, the classic 1948 descriptive caseseries by Philip Sandblom first used the term hemobilia to describe hemorrhage into the biliary tract after blunt trauma $[1 \bullet 2 \bullet]$. Historically, hemobilia has been associated with the triad of symptoms described by Heinrich Quincke in 1871 including pain, gastrointestinal bleeding, and jaundice. In practice, however, this triad is only present in $22 \%$ of the patients [1•]. Two recent reviews have summarized the evolution of diagnosis and management of hemobilia $[1 \bullet 3]$. We aim to provide a current update, focusing on common patient presentations and modern management strategies.

\section{Etiology}

The predominant etiology for hemobilia has changed over time. Historically, hemobilia was associated most commonly with external trauma, both penetrating and blunt. However, with the rise of percutaneous interventional techniques, iatrogenic hemobilia (i.e. after a procedure) is more frequent. Iatrogenic hemobilia currently comprises over $70 \%$ of all cases, with non-iatrogenic trauma considerably less frequent. Other causes of hemobilia are rare and include gallstones, malignancy, inflammatory diseases, and vascular etiologies $[1 \bullet, 3]$. The most frequent cause of hemobilia is percutaneous instrumentation of the biliary system including percutaneous biliary drainage, percutaneous transhepatic cholangiography, and needle liver 


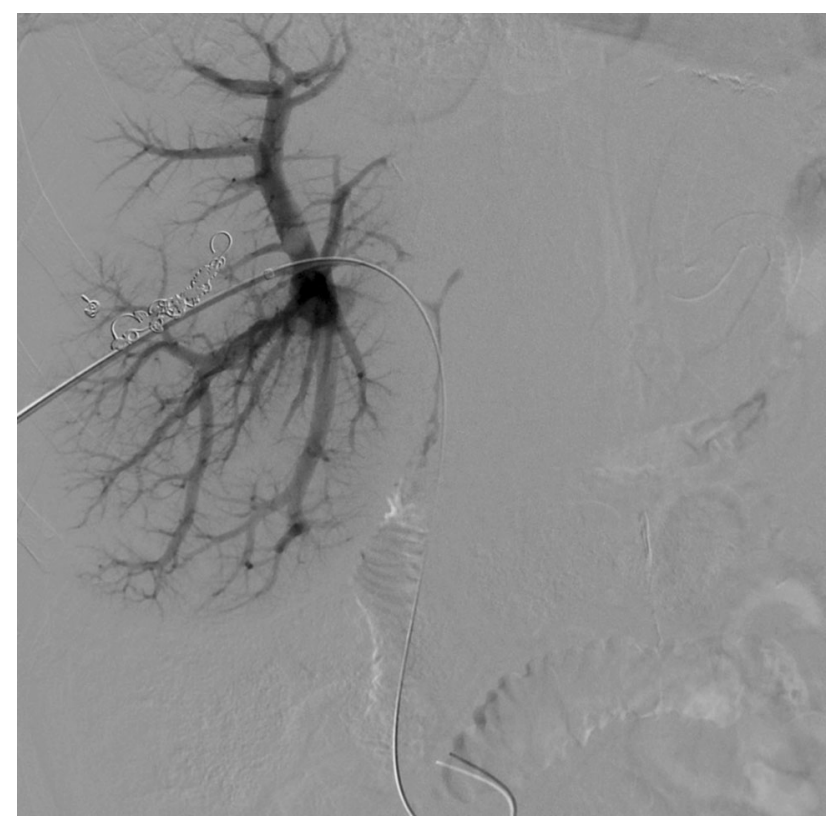

Fig. 1 Patient with venous hemobilia during percutaneous access managed with repositioning of the percutaneous transhepatic biliary drain

biopsy. Other biliary instrumentation including operative intervention can also cause iatrogenic post-operative hemobilia. Among patients in whom neither iatrogenic nor non-iatrogenic traumatic cause is evident, clinical evaluation should investigate intrahepatic or biliary malignancy (i.e. hepatocellular carcinoma, cholangiocarcinoma, secondary hepatic metastases), gallstone disease, acalculous biliary inflammation including parasitic disease, vascular malformations, and severe coagulopathy.

\section{Patient Presentation and Evaluation}

Patients with acute hemorrhage frequently present with symptomatic anemia. Gastrointestinal bleeding (either upper or lower) and/or blood in the percutaneous or operatively-placed drains in a patient with any history of hepatobiliary manipulation or trauma should prompt a rapid evaluation for clinical hemobilia. The time interval between operative intervention and bleeding can be prolonged, particularly in cases of ongoing infection or persistent foreign body, such as post-traumatic abscess requiring drainage or prolonged biliary drainage for recurrent infections or tumor obstruction. Pseudoaneurysms of hepatopancreatobiliary arterial arcades including hepatic, cystic, gastroduodenal, and supra- and infra-pancreatic arteries have been culprits in delayed hemobilia and frequently occur after iatrogenic operative or percutaneous injury, particularly in the setting of ongoing infection.
While an arterial source of hemorrhage is usually clinically apparent and symptomatic, venous hemobilia is rare. Pressure gradients in the hepatic venous and portal venous systems are usually low and the majority of fistulae between venous and biliary systems are asymptomatic. Patients with portal hypertension are an exception and bleeding must be aggressively managed in this population. When clinically evident, venous hemobilia is frequently associated with percutaneous transhepatic biliary drains, and it can be controlled with the tamponade effect provided by upsizing the drainage catheter or changing the access route if bleeding occurs during initial access (Fig. 1) [4].

Clinically significant hemobilia is associated with a $94 \%$ positive predictive value for concomitant arterial injury found during angiography [5]. As such, angiography remains the preferred diagnostic and management approach for patients with clinical hemobilia. Other diagnostic and imaging modalities, such as endoscopy, ultrasound, or cross-sectional imaging (CT and CT angiography in most cases), can be used in cases with low clinical suspicion. Multiple manuscripts have addressed various diagnostic tools and their roles in diagnosis of hemobilia $[1 \bullet, 3]$. Endoscopy can identify blood from the ampulla, but the sensitivity is low. Rarely, hemosuccus pancreaticus can present with trans-ampullary bleeding and the diagnosis should remain in the differential, particularly among patients with pancreatitis [6]. Ultrasound and cross-sectional imaging can suggest clotted blood or demonstrate evidence of ongoing bleeding. The specificity of these imaging modalities is low and non-angiography imaging should not be used in instances of high clinical suspicion. Nuclear medicine imaging in evaluation and management of hemobilia is largely of historic significance.

Initial management of a patient with clinical suspicion for hemobilia must begin with appropriate goal-directed resuscitation and correction of coagulopathy. In hemodynamically stable patients, CT angiography can be a useful initial study. The sensitivity and specificity of modern CT angiography are high, allowing identification of the bleeding source for appropriate targeted therapy. Importantly, however, when there is a high suspicion for hemobilia, percutaneous angiography by an experienced hepatopancreatobiliary interventional radiologist is the initial study of choice, as it is critical both for establishing the diagnosis of hemobilia and treating the underlying cause of bleeding.

\section{Iatrogenic Hemobilia}

Currently, over $70 \%$ of cases of hemobilia result from hepatobiliary instrumentation. Risk of hemobilia after percutaneous transhepatic biliary drainage (PTBD) is 
approximately $2 \%$, while the risk of hemobilia after percutaneous transhepatic cholangiography (PTC) without placement of a drain is less than $1 \%$ [5]. Hemobilia after a liver biopsy alone is considerably more rare [7]. Endoscopic trans-ampullary access and instrumentation can cause transient and frequently asymptomatic hemobilia. Post-ERC bleeding usually resolves with non-interventional management; however, adequate biliary drainage must be confirmed [8].

Hemobilia after hepatobiliary resection is rare. Its occurrence is associated with other biliary complications, such as biliary leak or biloma. Similar to the vascular bleeding complications after pancreaticoduodenectomy, clinically significant hemobilia can occur when a postoperative organ space infection develops in the presence of a disrupted or reconstructed biliary tree and surgically altered vascular system or an unrecognized injury to the hepatic vasculature. Pseudoaneurysms of the right hepatic artery and/or cystic artery were observed in the early laparoscopic experience [9]. While combined vasculobiliary injuries and isolated vascular injuries are important, they are relatively rare current complications of laparoscopic cholecystectomy [10]. The rate of hemobilia and/or pseudoaneurysm formation among patients with minimally invasive hepatic resections has not been well described nor compared to open resection data.

Liver transplant recipients, particularly living-donor liver transplant recipients, and patients transplanted after neoadjuvant chemoradiation for cholangiocarcinoma, are at risk of vasculobiliary complications [11]. Hepatic arterial and biliary system reconstructions are in close proximity. Biliary leak and/or post-transplant biloma can undermine the arterial anastomosis and lead to a pseudoaneurysm formation. While extremely rare, these complications usually require hepatic artery revision and occasionally retransplantation. Percutaneous embolization and/or intraluminal stent placement have been described, but both can lead to thrombosis, dissection, or arterial rupture with subsequent need for re-transplantation.

\section{Traumatic Hemobilia}

Blunt liver trauma is common. Currently, the majority of blunt liver injuries are managed non-operatively [12]. Goal-directed resuscitation and correction of the lethal triad of acidosis, hypothermia, and coagulopathy are critical in the early management of blunt liver injury. Despite early skepticism, there are no data to support more frequent biliary complications, including hemobilia, among trauma patients treated non-operatively [13]. Abdominal exploration with operative management of hepatic injury is frequently utilized in patients with penetrating liver trauma
[14, 15]. Non-operative management of post-traumatic hemobilia with endovascular techniques is safe and effective regardless of the initial mechanism of injury or choice of management $[16,17]$.

\section{Malignancy and Inflammatory Disease}

Numerous case reports and series describe hemobilia in patients with advanced primary and secondary hepatobiliary malignancies. The majority of these patients present with advanced tumor stage and are not candidates for resection. Hemobilia is also a rare manifestation of advanced benign inflammatory pancreatobiliary disease with or without the presence of gallstones. Management of hemobilia and biliary drainage should precede cholecystectomy in patients with suspected cholecystitis. In addition, parasitic infections, such as Echinococcus, Clonorchis, and Ascaris, have all been associated with hemobilia $[1 \bullet, 3]$.

\section{Management}

While hemobilia often results in surgical consultation, it rarely requires operative intervention. Treatment relies almost universally on angiography and percutaneous endovascular techniques. Arterial bleeding is episodic and often does not respond to resuscitation without intervention. Fortunately, percutaneous arterial embolization is highly effective in identifying and controlling arterial bleeding [18]. With advances in endovascular treatment options, previously reported treatment failures [19] can be managed successfully with evolving transcatheter technology and growing clinical experience. Success of endovascular management at experienced centers approaches $100 \%$ [5, 20]. Re-operation for a rare failure of trans-catheter therapy is a formidable task and should be performed at institutions with hepatobiliary expertise. Operative management varies, depending on the cause for persistent hemobilia, but almost always involves ligation of the bleeding vessel (most frequently the right hepatic artery) and drainage of the biliary system.

Part of the reason for the reported success of arterial embolization in controlling arterial-biliary bleeding is the dual blood supply to the liver which allows aggressive occlusion of peripheral arterial branches without a high risk of subsequent liver ischemia or infarction. Trans-arterial delivery of coils to an injured artery prevents further bleeding but also occludes the branch. The goal of embolization is always to preserve as much hepatic artery as 
Fig. 2 Patient with pseudoaneurysm of the proximal right hepatic artery (a) managed with trans-arterial coil embolization (b)
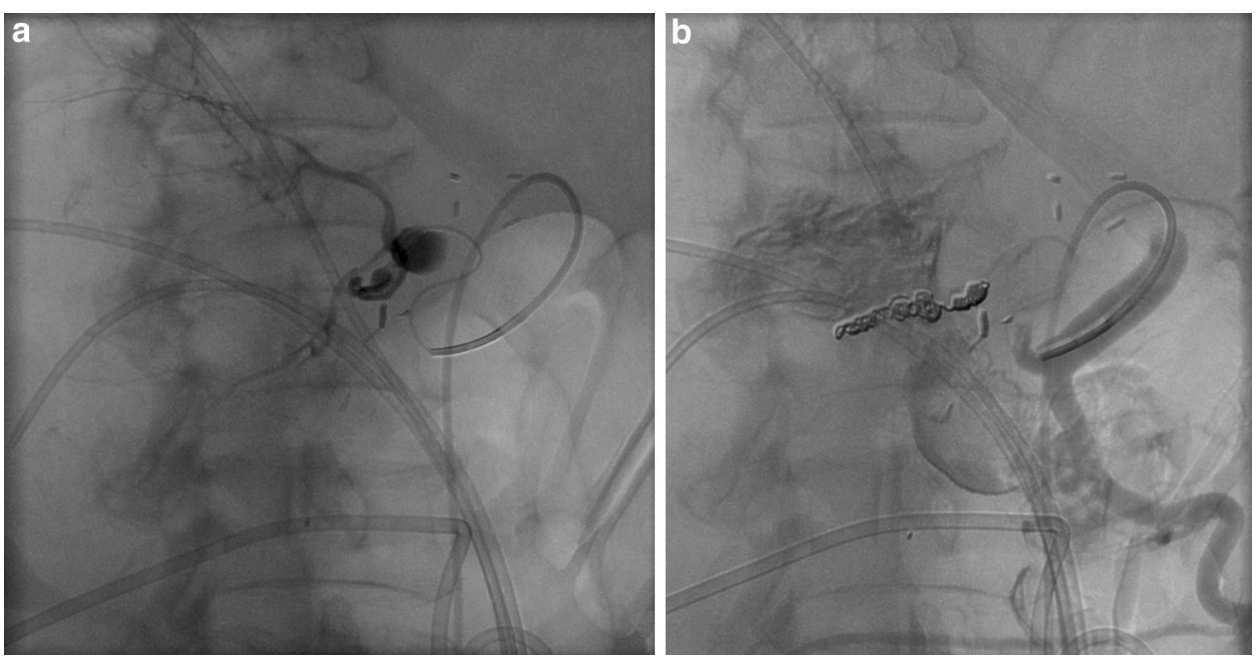

possible, but embolization of the injured artery both proximal and distal to the site of injury are important to prevent recurrent bleeding (Fig. 2).

Most cases of iatrogenic hemobilia are due to intraparenchymal arterial-biliary communications. More proximal hepatic artery injury in the hepatic hilum can also cause hemobilia. Although collateral flow should in theory preserve the liver, proximal embolization is more problematic in post-operative patients and patients in shock [21]. Significant hepatic injury or liver failure can occur with common or proper hepatic artery embolization. There is no test to predict the likelihood of liver ischemia after proximal embolization, but the clinical scenario can indicate high-risk situations. Liver transplants are particularly high risk due to the lack of collaterals and the more frequent complication of biliary necrosis after proximal embolization [22].

Covered stents have enabled percutaneous endovascular control of bleeding common or proper hepatic artery pseudoaneurysms, while still preserving hepatic artery flow [23]. These covered stents often permanently exclude the leak, and preserve the lumen at least in the short term. Their long-term durability remains a question due to intimal hyperplasia and associated stent stenosis and possible thrombosis [24].

Biliary drainage is imperative to successful management of hemobilia. While hemorrhage cessation is the primary therapeutic goal, the majority of the patients present with concomitant biliary obstruction from intraluminal bleeding and clot. Endoscopic management is the preferred initial strategy for decompression of the biliary tree [25]. Patients with biliary obstruction who are not candidates for endoscopic management require PTBD. Since the vascular and biliary systems are both affected in patients with hemobilia, interventions directed at both are necessary for optimal patient outcome.

\section{Conclusions}

Hemobilia remains a rare cause for acute hemorrhage and biliary obstruction. Hemobilia must be suspected in all patients with a recent history of biliary instrumentation or operative intervention. Gastrointestinal bleeding and/or extraluminal blood in surgical or percutaneous drains in a patient with recent hepatobiliary instrumentation, operation, trauma, or any known pathology should be evaluated for presumed hemobilia. CT angiography can be the initial diagnostic evaluation in a hemodynamically stable patient; however, angiography with trans-catheter management is the standard diagnostic and treatment approach in patients with clinical hemobilia. Appropriate biliary drainage must be ensured after bleeding is controlled.

\section{Compliance with Ethics Guidelines}

Conflict of Interest Victor Zaydfudim declares that he has no conflict of interest. John Angle has served as a consultant for Terumo Medical and Medtronic and has received grant support from Siemens Medical. Reid Adams declares that he has no conflict of interest.

Human and Animal Rights and Informed Consent This article does not contain any studies with human or animal subjects performed by any of the authors.

\section{References}

Recently published papers of particular interest have been highlighted as:

- Of importance

1. - Green MH, Duell RM, Johnson CD, Jamieson NV. Haemobilia. Br J Surg. 2001;88:773-86. Comprehensive summary of caseseries reports on presentation, etiology, evaluation, and management of hemobilia. 
2. - Sandblom P. Hemorrhage into the biliary tract following trauma; traumatic hemobilia. Surgery. 1948;24:571-86. Original case-series describing hemobilia. Study of historic interest and importance.

3. Chin MW, Enns R. Hemobilia. Curr Gastroenterol Rep. 2010;12:121-9.

4. Winick AB, Waybill PN, Venbrux AC. Complications of percutaneous transhepatic biliary interventions. Tech Vasc Interv Radiol. 2001;4:200-6.

5. Fidelman N, Bloom AI, Kerlan RK Jr, et al. Hepatic arterial injuries after percutaneous biliary interventions in the era of laparoscopic surgery and liver transplantation: experience with 930 patients. Radiology. 2008;247:880-6.

6. Sakorafas GH, Sarr MG, Farley DR, Que FG, Andrews JC, Farnell MB. Hemosuccus pancreaticus complicating chronic pancreatitis: an obscure cause of upper gastrointestinal bleeding. Langenbecks Arch Surg. 2000;385:124-8.

7. Piccinino F, Sagnelli E, Pasquale G, Giusti G. Complications following percutaneous liver biopsy. A multicentre retrospective study on 68,276 biopsies. J Hepatol. 1986;2:165-73.

8. Baron TH. Hemobilia-induced occlusion of a biliary metal expandable stent. Gastrointest Endosc. 2001;54:362.

9. Nicholson T, Travis S, Ettles D, et al. Hepatic artery angiography and embolization for hemobilia following laparoscopic cholecystectomy. Cardiovasc Intervent Radiol. 1999;22:20-4.

10. Strasberg SM, Helton WS. An analytical review of vasculobiliary injury in laparoscopic and open cholecystectomy. HPB (Oxford). 2011;13:1-14.

11. Mantel HT, Rosen CB, Heimbach JK, et al. Vascular complications after orthotopic liver transplantation after neoadjuvant therapy for hilar cholangiocarcinoma. Liver Transpl. 2007;13:1372-81.

12. Parks RW, Chrysos E, Diamond T. Management of liver trauma. Br J Surg. 1999;86:1121-35.

13. Bynoe RP, Bell RM, Miles WS, Close TP, Ross MA, Fine JG. Complications of nonoperative management of blunt hepatic injuries. J Trauma. 1992;32:308-14 discussion 14-5.
14. Badger SA, Barclay R, Campbell P, Mole DJ, Diamond T. Management of liver trauma. World J Surg. 2009;33:2522-37.

15. Zaydfudim V, Dutton WD, Feurer ID, Au BK, Pinson CW, Cotton BA. Exsanguination protocol improves survival after major hepatic trauma. Injury. 2010;41:30-4.

16. Forlee MV, Krige JE, Welman CJ, Beningfield SJ. Haemobilia after penetrating and blunt liver injury: treatment with selective hepatic artery embolisation. Injury. 2004;35:23-8.

17. Moodley J, Singh B, Lalloo S, Pershad S, Robbs JV. Non-operative management of haemobilia. Br J Surg. 2001;88:1073-6.

18. Saad WE, Davies MG, Darcy MD. Management of bleeding after percutaneous transhepatic cholangiography or transhepatic biliary drain placement. Tech Vasc Interv Radiol. 2008;11:60-71.

19. Dousset B, Sauvanet A, Bardou M, Legmann P, Vilgrain V, Belghiti J. Selective surgical indications for iatrogenic hemobilia. Surgery. 1997;121:37-41.

20. Defreyne L, Vanlangenhove P, De Vos M, et al. Embolization as a first approach with endoscopically unmanageable acute nonvariceal gastrointestinal hemorrhage. Radiology. 2001;218:739-48.

21. Nagaraja R, Govindasamy M, Varma V, et al. Hepatic artery pseudoaneurysms: a single-center experience. Ann Vasc Surg. 2013;27:743-9.

22. Saad WE. Management of nonocclusive hepatic artery complications after liver transplantation. Tech Vasc Interv Radiol. 2007; 10:221-32.

23. Krokidis ME, Hatzidakis AA. Acute hemobilia after bilioplasty due to hepatic artery pseudoaneurysm: treatment with an ePTFEcovered stent. Cardiovasc Intervent Radiol. 2009;32:605-7.

24. Hankins D, Chao S, Dolmatch BL, Jeyarajah RD. Covered stents for late postoperative arterial hemorrhage after pancreaticoduodenectomy. J Vasc Interv Radiol. 2009;20:407-9.

25. Kim KH, Kim TN. Etiology, clinical features, and endoscopic management of hemobilia: a retrospective analysis of 37 cases. Korean J Gastroenterol. 2012;59:296-302. 